\title{
COVID-19 Pandemic and Research Publications; Necessity of Maintaining Scientific Integrity
}

\author{
D. Qaiser
}

Editor, International Annals of Science

Corresponding author email: qaiser.d@gmail.com

Received: 01 September 2020 / Revised: 04 September 2020 / Accepted: 04 September 2020 / Published: 04 September 2020

\begin{abstract}
Since the COVID-19 outbreak began in China, scientists and health professionals have rushed to understand and mitigate the threat, however, its root cause, spreading characteristic, effective way to control as well as therapeutical approaches are still a mystery and matter of scientific debate. In an effort to fight against this disease scientists also rushed for a global collaborative approach by sharing their findings so that others can use known information. In view of such emergency scientific journals took steps to expedite the peer review process for coronavirus-related manuscripts which poses another challenge of scientific integrity. Community attention on integrity brought another concern where many authors argue against the idea of peer-review exception which compromises high standard for quality in the name of crisis situations. In the space of eight months, the research community's response to COVID-19 gives rise to a large volume of paper submission which required rigorous reviewing and of course huge amount of time however, it's also time which demands fastest publication of the latest finding. To balance in scientific integrity of scholarly journal as well as crisis demand to expedite dissemination of known knowledge, AIJR took a unique approach for COVID-19 related manuscript submission to Int. Ann. Sci. only through AIJR preprints invitation. In this approach author shall post COVID-19 related articles to AIJR Preprints and if it seems suitable for further peer-reviewing, author will get invited to submit to Int. Ann Sci. otherwise author will be advised to improve the article with an updated version. In this way the latest finding can get disseminated immediately as a preprint and after submission to the journal it can undergo standard reviewing process to maintain the scientific integrity. Although invitation through preprints serves both purpose of fastest dissemination and the journal can maintain scientific integrity, preprint may have its own risk for sharing non-reviewed version which may include dishonest findings. The only way to make preprints a great place for accelerated publishing and minimize associated risk of sharing non-reviewed findings is that the authors, readers, and most importantly media reporters act in a vigilant manner by following the sharing responsibility and guidelines adhering to the highest ethical standards.
\end{abstract}

Keywords: Editorial, Scientific Integrity, Preprint

\section{A Glimpse of COVID-19}

COVID-19 outbreak began from Wuhan, China at the end of 2019 with a disease similar to severe acute respiratory syndrome (SARS). Many of the initial cases for which scientists considered as a new coronavirus spreading to humans were linked to a live market in the Wuhan city. Within the weeks of initial cases reported, researchers were successfully isolate and sequence the virus's RNA code. Fan Wu et al. [1] alerted the world on 12 January 2020 for the similarity of this new coronavirus with SARS-CoV-1 by releasing the genome sequence into GenBank database [2] which was isolated from a single patient who had worked at the Wuhan live animal market.

Now in September 2020, approx. eight months later, the worldwide scientists have released nearly 18500 Nucleotide \& more than 191,400 Protein sequence related to SARS-CoV-2 [3]. This vital collection of genetic information has allowed researchers to describe the different observed severity level in different regions 
however, a satisfactory reason for alternation in the virus characteristic and its means for transmission as well as disease is not yet very clear [4].

Initial reports of the disease characterized as a severe respiratory illness similar to that caused by SARS-CoV-1 which could be transmitted from person to person either through direct contact or indirect, such as through a simple exhalation or through droplets expelled during a cough [4]. As the virus began to spread around the world, data from the Diamond Princess cruise ship and other studies [5], [6] revealed that people can transmit the virus asymptotically even if they are not sick, which is probably contributing to its rapid spreading.

Soon it became apparent that SARS-CoV-2 is not just a respiratory virus but exists in other organs than the lungs, including the respiratory tract, heart, kidneys, liver, and brain, even in the blood [7]. The SARS-CoV-2 is found to be playing in different ways from place to place and even from person to person. Symptoms experienced by different persons found differently that may be attributed to their existing health condition in combination with the type of infected organ's cells and tissues by the virus. For instance, severely ill patients with an existing chronic disease show hyperactivation of their immune response, which damages their lungs [4]. But most important question that what causes the sudden onset of acute respiratory distress in some patient is still unknown.

Scientists are still struggling to corelate and understand the factors behind the person to person varying severity level which may lead to an effective treatment. Significant progress has been seen in the treatment of severe COVID-19 disease using the steroid drug dexamethasone, which suppress the overactive immune response [8]. Prior to the dexamethasone trial, the antiviral drug Remdesivir was the only treatments that showed to combat COVID-19 in rigorous clinical trials [9]. Convalescent plasma therapy is being used in many countries including India as pilot study and it's the recent hope as it holds good science behind its possibility to work as an effective treatment. However, scientist shows concern over plasma therapy as it has been tested only in small trials [10].

In an effort to understand that whether people who developed antibodies against SARS-CoV-2 and recovered are protected from reinfection or not as well as to know that how long those antibodies persist in the body, scientists in Iceland found that the antibodies against SARSCoV-2 did not decline within 4 months after diagnosis [11]. This finding countering the earlier evidences which suggested that these antibodies disappears quickly. It seems that as soon as scientists develop some understanding, new research data poses a new challenge by countering the previous findings. Scientists are working toward getting enough data and trials in order to understand the virus and develop medicine for an effective treatment however it's too early to conclude anything firmly.

\section{COVID-19 Rapid Publication versus Integrity}

As the global coronavirus pandemic evolves, scientists worldwide are conducting studies to address the crisis at unprecedented rates. Many publishers expedites their peer-reviewing process for COVID-19 publishing [12] in response to rapid dissemination requirement of latest finding related to the ongoing pandemic. Scientific integrity in a publication being maintained by scholarly journals through rigorous peer-review process [13] which requires plenty of time. Rapidreviewing demands great measure to safeguard the scientific integrity $\&$ trust in the publication [14]. Therefore, quick peer-reviewing should only be offered when scientific integrity can be safeguard, failing which may lead to devastating consequences for the public health. However, London and Kimmelman are against such exceptionalism of quick review and I also agree with their argue that the problem for which rigorous scientific methods are designed to identify through peer-reviewing process [13] shall not be ignored in the face of urgency [15].

The result of rapid publication can be seen as a rise in low-quality studies some of these studies have caught the public's attention. A study on COVID-19 publications through PubMed search found that the majority of early COVID-19 
D Qaiser, Int. Ann. Sci.; Vol. 10, Issue 1, pp: 1-6, 2021

articles provide no new information on the disease [16]. This study concluded that such publication actually slowing down our understanding of the disease as original findings on the COVID-19 pandemic are being crowded out by reviews and opinion articles. Scientists worrying the integrity in scholarly publications argue that the research quality should not be compromised through exceptions simply because of the COVID-19 pandemic [15] and the demand of rapid dissemination.

In a recent interview by CMAJ [17] on data quality concerns Dr. Matthew Oughton suggest authors to avoid predatory journals however, he didn't mention about reputed journals where such concerns are more serious than predatory ones as evident from Retraction Watch data [18] on COVID-19 related retraction trend. Further, Dr. Matthew also suggest editors for allowing reviewers sufficient time to thoroughly evaluate manuscripts which indicates that he is not in favor of rushed reviewing process at the cost of its quality.

In view of recent concern over scientific integrity The European Network of Research Integrity Offices (ENRIO) issued statement that research integrity even more important for research during a pandemic [19]. ENRIO reminded that the scholarly community has established rules for robust, peer-reviewed and trustworthy research. Failing to which will have a negative impact on research: They highlighted that bad practices will obstruct our efforts to stop the pandemic and save lives due to misrepresentation of our understanding about COVID-19 [19]. ENRIO noted that researchers should communicate their work responsibly and urged the research community to respect the highest integrity standards in research with professionalism and transparency for the benefit of humanity.

\section{Concern over COVID-19 Publications}

COVID-19 has a profound impact on the research community as well as scholarly journals. In response to the COVID-19 emergency, scholarly journals have been overwhelmed with publication, however, most of them are in short format and observational research. Presses and social media acting as amplifier to spread the
COVID-19 developments, often without distinguishing between fake science and solid science which is unprecedented in the history of modern medicine [20]. Not only lay media but also scholarly and even reputed journals publishes questionable COVID-19 papers, some of them got public attention and been retracted. As a result Retraction Watch noticed that such work is being retracted at higher rates than the rest of the literature [18].

Such journals where the time between submission and acceptance of mostly articles are only week or less characterized as predatory however, recent study of COVID-19 publication on PubMed search indicates that the average publication time was only 6 days [14] for even reputed journal. A paper representing the large number of studied patients regarding the use of hydroxychloroquine and chloroquine for the treatment for COVID-19 [21] becomes most controversial as it influenced the worldwide approach on the COVID-19 treatment and later this study itself found questionable and eventually retracted by LANCET [21]. Similarly, a paper got place in scholarly journal [22] which already been retracted after the community reaction, was considered as worst as it should not find a place even in predatory publications. Therefore, it is obvious that the peer review process has been weakened in the name of COVID-19 emergency even for the reputed journals [18]. It indicates that the emergency not only ignited the robust research but also the dishonest research reporting at an unprecedented rate. Ricardo termed it as a 'paperdemic' [23] and warned that it will be even more complicated to manage if not properly scrutinized within time. Like others [20], Int. Ann. Sci. also striving to detect research misconduct and violation of publication ethics. A balance is always needed between the benefits of the rapid dissemination to new research and the threat to scientific integrity. Although AIJR already restricted COVID-19 related manuscript submission through AIJR Preprints invitation only [24], still in view of the current alarming situation, it is important that scholarly journals remain vigilant to assure the quality. International Annals of Science is trying to fulfil the need for quick and 
easy dissemination of new findings while maintaining the paper publishing quality through an approach of limiting COVID-19 related submission to AIJR Preprint's invitation only.

\section{Our Approach via AIJR Preprints}

One of the most inspiring things for research publications happened in the age of COVID-19 that the virus and the pandemic researchers have embraced preprint servers. Preprint publications often use quick publication of author's version that have been encouraged by this journal with an approach of inviting option for only suitable COVID-19 related manuscript to peer-review in Int. Ann Sci. AIJR Preprints is a multidisciplinary preprint server where authors may upload research work that will be published immediately as non-reviewed version for general readership. Authors by tradition benefit from the discussion as open review via reader comments, while the community benefits from early access to ongoing research work. Authors are required to submit a properly formatted manuscripts that undergoes a brief screening for technical formatting and its suitability. Although assured feedback can't be guaranteed in preprint as it highly depends on being noticed by the research community and their interest to provide feedback. To increase the probability of appropriate feedback, it is highly recommended that author share the preprints to their networks and peers for a critique.

Preprint seems a great place for such pandemic emergency demand however, it has own drawback as evident from recent incident [25] where The Straits Times (a national newspaper in Singapore) reported the COVID-19 incubation period as to stretch out to 24 days based on a preprint report, which later revealed that this result was based on a single case out of the 1099 cases. Subsequently the same manuscript published NEJM, where the reported data has changed to a median incubation period of 4 days [25]. In this digital era where information can be widely and quickly disseminated, it is important to do all possible effort in order to minimize the source of misinformation. A multimodal approach and caution require by the authors, readers and media in order to minimize misinformation by maintaining transparency in sharing and reporting as outlined below.

\subsection{Responsibility of Authors}

As members of the academic \& scientific community, authors shall respect the highest integrity standards in research and communicate their work responsibly for the benefit of humanity. In the pandemic era there is high level of public interest in COVID-19 findings and hence it's the most crucial time for authors to use their scientific methodology and writing ability while maintaining highest standard. Young researchers shall look repeatedly for the available ethics for authors from academic organizations and publisher's ethical policy. It is important to update the preprint as soon as new finding or changes to data are made. For better and complete transparency, the changes in the updated version of the manuscripts may be highlighted with an interpretation that what make authors to do such changes. While author reusing such information, they should clearly mention that information is based on the preprint article.

\subsection{Caution Requires for the Readers}

Readers requires to be more cautious in order to ensure that the information they are reading has been reviewed or not yet. It's easy, just look at the header of first page if it's a preprint will be clearly highlighted with text "NOT PEERREVIEWED" (normally at top right corner of header in AIJR Preprints). If readers share or referencing such results to the public (e.g. through social sharing or blog), they should clearly mention that the information is from a preprint article that has not been peer-reviewed.

\subsection{Responsibility of the Media}

Media shall report research work through experienced scientific journalists who understand the difference in a preprint server and scholarly journal. Media and other staffing reduction show lack of scientific journalist and as a consequence the communication gap between scientists and journalists widen. Reporters need to understand fundamentals of research reporting and the challenges inherent in spreading the scientific research to its audience. Before reporting such 
findings, reporters shall ensure whether its peerreviewed article or not by looking at the first page of the article which will mention "NOT PEER RVIEWED" or its variant in the case of a preprint and if so, they shall clearly mention that the report is based on a preprint article which is not yet been peer-reviewed.

\section{Conclusions}

The recent coronavirus SARS-CoV-2 and the disease it causes, COVID-19, have ignite the research work globally at an unprecedented rate. Many scholarly journals expedite their reviewing process in the response of emergency however the recent data shows that it weakens quality of the paper and in some cases dishonest research got successfully published in reputed journals which is seen as an alarming situation for the scientific integrity. Another matter of fact which supports the argument against the reviewing exception is that the most of the coronavirus experts are intensifying their own research programs in response to the outbreak, and hence quick peer-reviewing pressure on them may result in low quality critique. In this emergency pandemic era, scientific community must adhere to their ethical responsibility at the highest standard. To meet the demand of both, rapid publication and maintaining scientific integrity, publication through preprint becomes need of this pandemic emergency. This is the main reason that Int Ann. Sci is considering coronavirus related submission through AIJR Preprints server only. Even researchers have embraced preprint servers in this pandemic era however, caution required while sharing such findings by media and lay persons. Not only lay person and press but also insightful medically trained readers require to be vigilant and share information responsibly by acknowledging and indicating the outcome is non-reviewed in order to make preprint servers as a valuable resource.

\section{Competing Interests}

The author is an editorial board member of this journal.

\section{How to Cite this Article:}

D. Qaiser, "COVID-19 Pandemic and Research Publications; Necessity of Maintaining Scientific Integrity”, Int. Ann. Sci., vol. 10, no. 1, pp. 1-6, Sep. 2020.

\section{References}

[1] F. Wu et al., "A new coronavirus associated with human respiratory disease in China," Nature, vol. 579, no. 7798, pp. 265-269, Mar. 2020, doi: 10.1038/s41586-020-2008-3.

[2] "Severe acute respiratory syndrome coronavirus 2 isolate Wuhan-Hu-1, co - Nucleotide - NCBI." https://www.ncbi.nlm.nih.gov/nuccore/MN908947 (accessed Sep. 03, 2020).

[3] "Severe acute respiratory syndrome coronavirus 2 data hub," NCBI Virus, 2020. https://www.ncbi.nlm.nih.gov/labs/virus/vssi/\#/virus? SeqType_s=Nucleotide $\&$ VirusLineage_ss=Severe acute respiratory syndrome coronavirus 2 (SARS-CoV2), taxid:2697049 (accessed Sep. 01, 2020).

[4] "Progress report on the coronavirus pandemic.," Nature, vol. 584, no. 7821, p. 325, Aug. 2020, doi: 10.1038/d41586-020-02414-1.

[5] K. Mizumoto, K. Kagaya, A. Zarebski, and G. Chowell, "Estimating the asymptomatic proportion of coronavirus disease 2019 (COVID-19) cases on board the Diamond Princess cruise ship, Yokohama, Japan, 2020," Eurosurveillance, vol. 25, no. 10, p. 2000180, Mar. 2020, doi: 10.2807/15607917.ES.2020.25.10.2000180.

[6] X. He et al., "Author Correction: Temporal dynamics in viral shedding and transmissibility of COVID-19 (Nature Medicine, (2020), 26, 5, (672-675), 10.1038/s41591-020-0869-5)," Nature Medicine. Nature Research, pp. 1-3, Aug. 07, 2020, doi: 10.1038/s41591-020-1016-z.

[7] V. G. Puelles et al., "Multiorgan and Renal Tropism of SARS-CoV-2," The New England journal of medicine, vol. 383, no. 6. NLM (Medline), pp. 590-592, Aug. 06, 2020, doi: 10.1056/NEJMc2011400.

[8] H. Ledford, "Coronavirus breakthrough: dexamethasone is first drug shown to save lives," Nature, vol. 582, no. 7813. NLM (Medline), p. 469, Jun. 01, 2020, doi: 10.1038/d41586-020-01824-5.

[9] H. Ledford, "Hopes rise for coronavirus drug remdesivir," Nature, Apr. 2020, doi: 10.1038/d41586020-01295-8.

[10] H. Ledford, "Evidence lags behind excitement over blood plasma as a coronavirus treatment," Nature, Aug. 2020, doi: 10.1038/d41586-020-02324-2.

[11] D. F. Gudbjartsson et al., "Humoral Immune Response to SARS-CoV-2 in Iceland," N. Engl. J. Med., p. NEJMoa2026116, Sep. 2020, doi: 10.1056/NEJMoa2026116.

[12] J. Brock, "New initiative cuts initial peer review to days, not months, for coronavirus research | Nature Index," Nature Index, 2020. https://www.natureindex.com/news-blog/rapidregistered-report-coronavirus-aims-to-stopresearchers-following-false-research-leads (accessed Sep. 01, 2020).

[13] A. A. Bahishti, "Peer Review: Critical Process of a Scholarly Publication," J. Mod. Mater., vol. 2, no. 1, pp. 1.1-1.2, Oct. 2016, doi: 10.21467/jmm.2.1.1.1-1.2.

[14] A. Palayew, O. Norgaard, K. Safreed-Harmon, T. H. Andersen, L. N. Rasmussen, and J. V. Lazarus, "Pandemic publishing poses a new COVID-19 challenge," Nature Human Behaviour, vol. 4, no. 7. Nature Research, pp. 666-669, Jul. 01, 2020, doi: 10.1038/s41562-020-0911-0.

[15] A. J. London and J. Kimmelman, "Against pandemic research exceptionalism," Science (80-. )., vol. 368, no. 
COVID-19 Pandemic and Research Publications; Necessity of Maintaining Scientific Integrity

6490, pp. 476 LP - 477, May 2020, doi: 10.1126/science.abc1731.

[16] N. Di Girolamo and R. Meursinge Reynders, "Characteristics of scientific articles on COVID-19 published during the initial 3 months of the pandemic," Scientometrics, pp. 1-18, Jul. 2020, doi: 10.1007/s11192-020-03632-0.

[17] L. Vogel, "How can the scientific community ensure the integrity of COVID-19 research?," CMAJ: Canadian Medical Association journal = journal de l'Association medicale canadienne, vol. 192, no. 28. NLM (Medline), pp. E817-E818, Jul. 13, 2020, doi: 10.1503/cmaj.1095881.

[18] "Retracted coronavirus (COVID-19) papers https://retractionwatch.com/retracted-coronaviruscovid-19-papers/ (accessed Sep. 01, 2020).

[19] "ENRIO Statement: Research integrity even more important for research during a pandemic - ENRIO." http://www.enrio.eu/enrio-statement-research-

integrity-even-more-important-for-research-during-apandemic/ (accessed Sep. 01, 2020).

[20] G. Citerio et al., "Critical care journals during the COVID-19 pandemic: challenges and responsibilities," Intensive Care Medicine, vol. 46, no. 8. Springer, pp. 1521-1523, Aug. 01, 2020, doi: 10.1007/s00134-02006155-7.

[21] The Lancet Editors, "Expression of concern: Hydroxychloroquine or chloroquine with or without a macrolide for treatment of COVID-19: a multinational registry analysis," The Lancet, vol. 395, no. 10240. Lancet Publishing Group, p. e102, Jun. 13, 2020, doi: 10.1016/S0140-6736(20)31290-3.

[22] "Paper blaming COVID-19 on 5G technology withdrawn - Retraction Watch." https://retractionwatch.com/2020/07/26/paper-

blaming-covid-19-on-5g-technology-withdrawn/ (accessed Sep. 02, 2020)

[23] R. J. Dinis-Oliveira, "COVID-19 research: pandemic versus 'paperdemic', integrity, values and risks of the 'speed science," Forensic Sci. Res., vol. 5, no. 2, pp. 174-187, Apr. 2020, doi: 10.1080/20961790.2020.1767754.

[24] "Publishing Coronavirus Research Paper» Submit COVID-19 Articles." https://www.aijr.in/publishingcoronavirus-research-paper/ (accessed Sep. 04, 2020).

[25] N. Bagdasarian, G. B. Cross, and D. Fisher, "Rapid publications risk the integrity of science in the era of COVID-19," BMC Med., vol. 18, no. 1, p. 192, Jun. 2020, doi: 10.1186/s12916-020-01650-6.
Publish your research article in AIJR journals-

$\checkmark \quad$ Online Submission and Tracking

$\checkmark$ Peer-Reviewed

$\checkmark$ Rapid decision

$\checkmark \quad$ Immediate Publication after acceptance

$\checkmark \quad$ Articles freely available online

$\checkmark \quad$ Retain full copyright of your article.

Submit your article at journals.aijr.in

Publish your books with AIJR publisher-

$\checkmark \quad$ Publish with ISBN and DOI.

$\checkmark$ Publish Thesis/Dissertation as Monograph.

$\checkmark$ Publish Book Monograph.

$\checkmark \quad$ Publish Edited Volume/ Book.

$\checkmark$ Publish Conference Proceedings

$\checkmark \quad$ Retain full copyright of your books.

Submit your manuscript at books.aijr.org. 